\title{
The NAC-type transcription factor CaNAC46 regulates the salt and drought tolerance of transgenic Arabidopsis thaliana
}

Jing $\mathrm{Ma}^{\dagger}$, Li-yue Wang ${ }^{\dagger}$, Jia-xi Dai, Ying Wang and Duo Lin ${ }^{*}$

\begin{abstract}
Background: The NAC (NAM, ATAF1/ATAF2, and CUC2) transcription factors belong to a large family of plantspecific transcription factors in monocot and dicot species. These transcription factors regulate the expression of stress tolerance-related genes that protect plants from various abiotic stresses, including drought, salinity, and low temperatures.
\end{abstract}

Results: In this study, we identified the CaNAC46 transcription factor gene in Capsicum annuum. Its open reading frame was revealed to comprise 921 bp, encoding a protein consisting of 306 amino acids, with an isoelectric point of 6.96. A phylogenetic analysis indicated that CaNAC46 belongs to the ATAF subfamily. The expression of CaNAC46 was induced by heat, cold, high salt, drought, abscisic acid, salicylic acid, and methyl jasmonate treatments. Thus, CaNAC46 may be important for the resistance of dry pepper to abiotic stresses. A subcellular localization analysis confirmed that CaNAC46 is localized in the nucleus. The overexpression of CaNAC46 improved the tolerance of transgenic Arabidopsis thaliana plants to drought and salt stresses. The CaNAC46-overexpressing lines had longer roots and more lateral roots than wild-type lines under prolonged drought and high salt stress conditions. Additionally, CaNAC46 affected the accumulation of reactive oxygen species (ROS). Moreover, CaNAC46 promoted the expression of SOD, POD, RD29B, RD20, LDB18, ABI, IAA4, and P5CS. The malondialdehyde contents were higher in TRV2-CaNAC46 lines than in wild-type plants in response to drought and salt stresses. Furthermore, the expression levels of stress-responsive genes, such as ABA2, P5CS, DREB, RD22, CAT, and POD, were down-regulated in TRV2CaNAC46 plants.

Conclusions: Under saline and drought conditions, CaNAC46 is a positive regulator that activates ROS-scavenging enzymes and enhances root formation. The results of our study indicate CaNAC46 is a transcriptional regulator responsible for salinity and drought tolerance and suggest the abiotic stress-related gene regulatory mechanisms controlling this NAC transcription factor are conserved between A. thaliana and pepper.

Keywords: Pepper, NAC transcription factor, Stress tolerance, Transgenic plants

\footnotetext{
* Correspondence: linduo73@163.com

${ }^{\dagger}$ Jing Ma and Li-yue Wang contributed equally to this work.

College of Horticulture, Qingdao Agricultural University, Key Laboratory of

Horticultural Plant Genetic Improvement and Breeding of Qingdao, 700

Changcheng Road, Qingdao 266109, China
}

(c) The Author(s). 2020 Open Access This article is licensed under a Creative Commons Attribution 4.0 International License, which permits use, sharing, adaptation, distribution and reproduction in any medium or format, as long as you give appropriate credit to the original author(s) and the source, provide a link to the Creative Commons licence, and indicate if changes were made. The images or other third party material in this article are included in the article's Creative Commons licence, unless indicated otherwise in a credit line to the material. If material is not included in the article's Creative Commons licence and your intended use is not permitted by statutory regulation or exceeds the permitted use, you will need to obtain permission directly from the copyright holder. To view a copy of this licence, visit http://creativecommons.org/licenses/by/4.0/ The Creative Commons Public Domain Dedication waiver (http://creativecommons.org/publicdomain/zero/1.0/) applies to the data made available in this article, unless otherwise stated in a credit line to the data. 


\section{Background}

Pepper (Capsicum annuum; family: Solanaceae) is a popular vegetable crop cultivated worldwide. China is the largest producer and consumer of chili peppers, with approximately $40 \%$ of the global chili cultivation area [1]. In addition to being widely used as a condiment, dry peppers are also used in the medical and military industries [2]. However, there are some environmental conditions, such as salinization and drought, that adversely affect the main dry pepper-producing areas.

Abiotic stresses, including drought, high-salinity, and temperature extremes, are the main factors responsible for decreased dry pepper quality [3]. To protect against the detrimental effects of environmental stresses, plants have gradually evolved appropriate defense mechanisms, in which the regulation of gene expression plays an important role. Transcription factors are critical regulators of abiotic stress tolerance [4]. In plants, the large NAC (NAM, ATAF1/ATAF2, and CUC2) transcription factor family is involved in abiotic stress responses [5]. The N-terminal of NAC transcription factors is highly conserved, in contrast to the variable C-terminal [6]. This transcription factor family has recently been extensively studied in Arabidopsis thaliana, rice, tobacco, and soybean [710]. There has also been increasing interest in these transcription factors in pepper crops [11, 12]. A genome-wide analysis of 104 NAC transcription factors in hot pepper and an expression analysis of 22 NAC transcription factor genes revealed diverse responses to various stresses [13]. As a transcriptional activator of CaPLD4, CaNAC1 helps degrade phospholipids in green pepper in response to low temperatures [11]. The pepper CaNAC2 transcription level is reportedly considerably up-regulated by an abiotic stress treatment, but is down-regulated by osmotic stress and a salicylic acid (SA) treatment [14].

The CaNAC46 gene was identified during a search of a pepper cDNA library with A. thaliana AtNAC2/ ATAF1/ANAC002 (At1g01720) as the query [14]. The CaNAC46 transcription factor helps regulate responses to abiotic stresses (e.g., drought, salinity, and low temperatures) and phytohormones [e.g., abscisic acid (ABA), methyl jasmonate (MeJA), and SA]. We demonstrated that CaNAC46 positively regulates plant tolerance to drought and salinity stresses, and developed a model for the role of CaNAC46 in the abiotic stress response system [15]. The results may provide the basis for a comprehensive analysis of the function and regulatory network of CaNAC46 related to plant stress resistance, with potential implications for the application of CaNAC46 in future genetic engineering experiments.

\section{Results}

Identification of the ATAF subfamily gene CaNAC46 in pepper

The NAC transcription factors are involved in mediating plant responses to abiotic stresses [16, 17]. The CaNAC46 gene was isolated from C. annuum var. 'Qingnong No. 2'. The encoded protein comprised 306 amino acids, with a molecular weight of $34.94 \mathrm{kDa}$ and a pI of 6.96 (Additional file 1: Table S1). Additionally, the proportions of basic and acidic amino acids were similar between CaNAC46 and the homologous NAC proteins in other model plants. There were also similarities in the other examined physical and chemical characteristics between CaNAC46 and its homologs in other model plants. These results suggest that NAC transcription factors have a conserved secondary structure.

In this study, CaNAC46 was clustered with ATAF1 [18], OsNAC5 [19], and OsNAC6 [20], which belong to the ATAF subfamily (Fig. 1A). A multiple sequence alignment revealed a sequence identity of $71.19 \%$, implying that the NAC transcription factor sequences were highly conserved. The CaNAC46 C-terminal contains the EVQS $[\mathrm{E} / \mathrm{x}] \mathrm{PK}[\mathrm{W} / \mathrm{I}]$ sequence, which is consistent with the sequences of typical ATAF subfamily members [3]. A phylogenetic analysis revealed that CaNAC46 is most closely related to OsNAC5, OsNAC6, and ANAC2 of the ATAF subgroup (Fig. 1B).

\section{CaNAC46 is localized in the nucleus}

An analysis of the fluorescent signals for the green fluorescent protein (GFP) alone (control) and the CaNAC46GFP fusion protein in tobacco cells with a confocal microscope indicated CaNAC46 is a nuclear protein (Fig. 2A).

\section{CaNAC46 expression is induced by abiotic stresses and phytohormones}

We examined the CaNAC46 expression level in response to various abiotic stresses over a 12 -h treatment period. The expression of CaNAC46 was significantly upregulated following drought, salt, heat, and cold stress treatments, especially from the 6-h time-point, with peak expression levels at $12 \mathrm{~h}$ (Fig. 2B). Additionally, CaNAC46 expression was also substantially induced by ABA, which is a phytohormone involved in salt and drought stress responses.

\section{Overexpression of CaNAC46 enhances the drought and salinity tolerance of transgenic Arabidopsis thaliana}

The effect of CaNAC46 overexpression on drought stress tolerance was investigated using transgenic plants treated with polyethylene glycol (PEG 6000). To assess the effect of CaNAC46 overexpression on salt stress tolerance, wild-type (WT) and transgenic seedlings were 


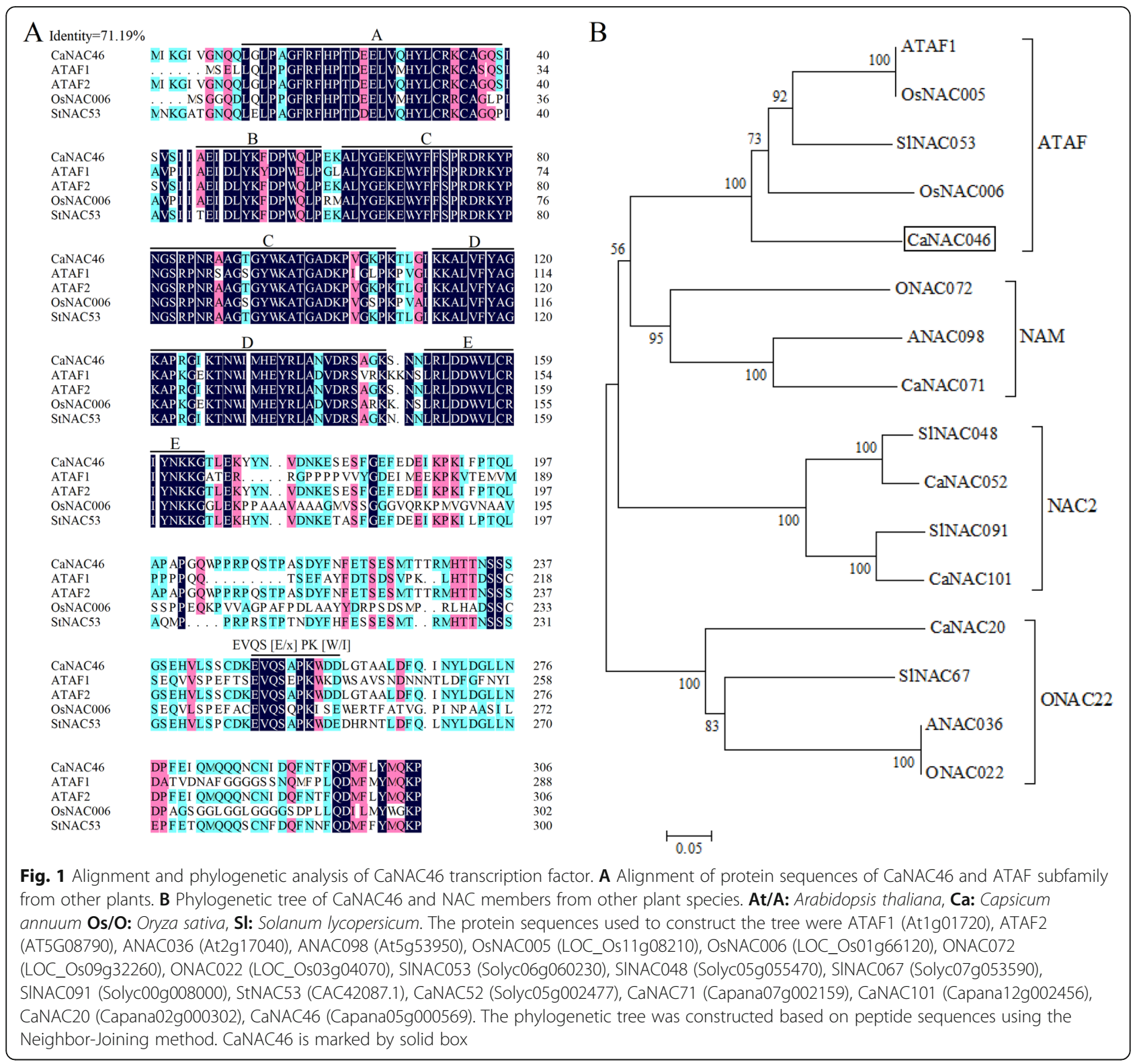

grown on 1/2 MS agar medium supplemented with 200 $\mathrm{mM} \mathrm{NaCl}$ (Fig. 3A). Under normal conditions, the germination rates of the WT and transgenic plants were approximately 95\%. However, the germination rates under the simulated drought and salt stress conditions differed substantially between the WT and CaNAC46overexpressing plants (Additional file 2: Figure S1).

The salinity and drought tolerance of the CaNAC46overexpressing lines was further evaluated at the vegetative growth stage. Following the drought and high salt treatments, the leaves of the WT plants were severely wilted, but the leaves of the CaNAC46-overexpressing plants were not (Fig. 3B). And after dehydration treatments, plants were re-watered 3 days, the leaves of WT remained wilted, while the $\mathrm{OE}$ lines grown normally (Fig. 3B).

\section{CaNAC46 overexpression promotes the root growth of transgenic Arabidopsis thaliana}

Plant root and shoot systems modify signaling and metabolic pathways to adapt to complex environmental changes and maintain normal growth and development [21]. A developed root system is important for improving plant stress resistance. In the current study, the $\mathrm{NaCl}$ treatment inhibited the lateral root development of WT seedlings. In contrast, the root elongation of two analyzed CaNAC46-overexpressing lines was only slightly inhibited, ultimately resulting in more lateral roots on 


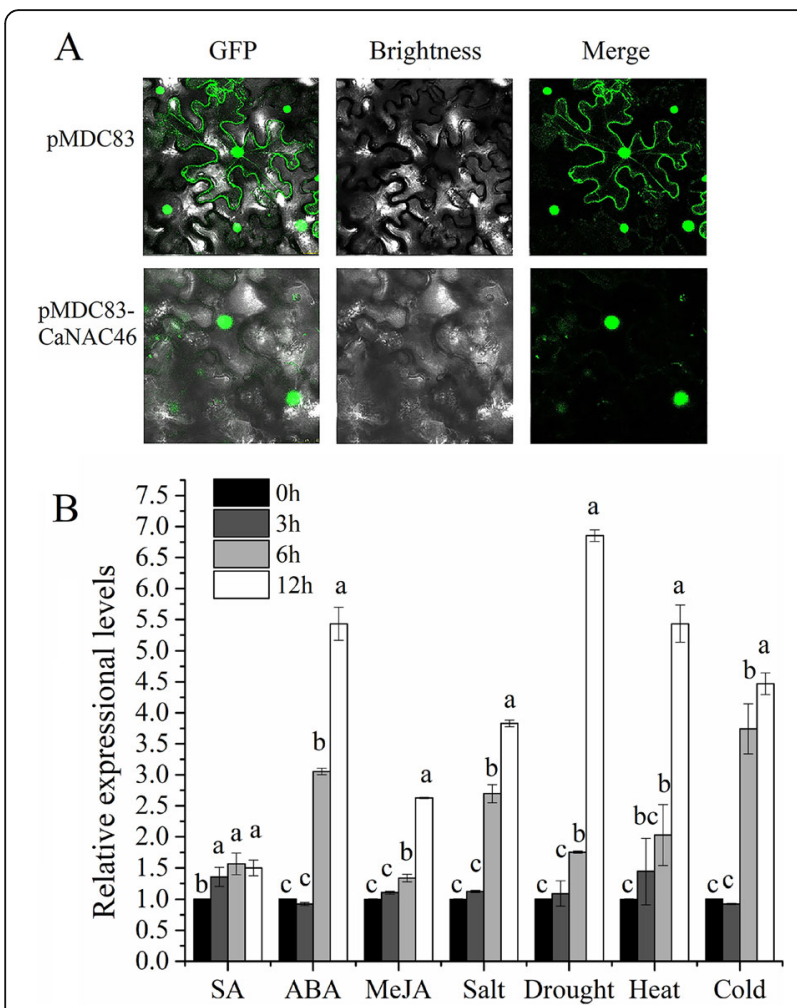

Fig. 2 Subcellular Localization and Stress-Responsive Expression. A Nuclear localization of CaNAC46 in tobacco cells. B Expression patterns of CaNAC46 in response to stress treatments. Superscript letters indicate significant differences. Three independent biological experiments were performed $(P<0.05)$

the transgenic plants than on the WT plants (Fig. 3A and $\mathrm{C}$ ). These observations implied that the overexpression of CaNAC46 may enhance abiotic stress resistance by promoting root growth (Fig. 3). Moreover, the expression levels of $L B D 18$ and $I A A 4$, which are involved in lateral root development, were significantly upregulated in the CaNAC46-overexpressing plants under drought and saline conditions (Fig. 4B).

\section{CaNAC46 overexpression decreases reactive oxygen species accumulation}

High CaNAC46 expression levels in the CaNAC46-overexpressing $A$. thaliana lines were confirmed by quantitative real-time PCR (qRT-PCR) (Additional file 3: Figure S2). Drought and salinity stresses usually induce the excessive accumulation of reactive oxygen species (ROS), which eventually leads to irreversible damages. We measured the SOD, POD, and CAT activities in the WT and CaNAC46-overexpressing plants under normal and stress conditions (Fig. 4A). There were no severe differences in the enzyme activities between the WT and transgenic plants under normal conditions. However, exposures to drought and salt stresses resulted in considerable increases in the SOD, POD, and CAT activities in both the WT and CaNAC46-overexpressing plants, but the increases were more pronounced in the CaNAC46overexpressing plants.

\section{CaNAC46 overexpression activates the expression of stress-related genes}

To clarify the putative regulatory effect of CaNAC46 on abiotic stress tolerance, qRT-PCR analyses were completed to examine the expression patterns of stressresponsive genes (e.g., $A B I, P 5 C S 1, R D 29 B$, and $R D 20$ ) in the WT and CaNAC46-overexpressing plants before and after the abiotic stress treatments (Fig. 4B). We observed that a relatively low CaNAC46 expression level impaired the water-deficit response of whole plants and detached leaves. In contrast, a relatively high CaNAC46 expression level protected plant from the damages due to abiotic stresses.

\section{Silencing of CaNAC46 decreases plant tolerance to high salt and drought conditions}

An analysis of the CaNAC46 expression levels in the CaNAC46-silenced (TRV2-CaNAC46) and WT plants before the stress treatments confirmed that CaNAC46 expression was significantly down-regulated in the TRV2-CaNAC46 lines (Additional file 4: Figure S3). The TRV2-CaNAC46 and WT plants were then subjected to drought stress (i.e., dehydrated) and salinity stress (i.e., treated with a $200 \mathrm{mM} \mathrm{NaCl}$ solution). The TRV2CaNAC46 lines showed a more weaker growth state than that of none-silenced lines under both normal and stress conditions (Fig. 5A). The cellular $\mathrm{H}_{2} \mathrm{O}_{2}$ level determined by diaminobenzidine (DAB) staining was higher in the TRV2-CaNAC46 leaves than in the WT leaves after 3-week salt and drought treatments (Fig. 5B). Additionally, the malondialdehyde (MDA) content, which may be used as an indicator of lipid peroxidation, increased considerably in the TRV2-CaNAC46 plants, but not in the WT plants, during the later stages of the drought and salinity treatments (Fig. 5C). Moreover, in the TRV2-CaNAC46 plants, CAT6 (encoding a catalase important for plant cell responses to stress), PR1, and $R D 22$ expression levels were down-regulated and the ROS-scavenging activity was low, which increased the sensitivity of these plants to saline and drought conditions (Fig. 5D).

\section{Discussion}

Diverse stresses adversely affect the growth and productivity of dry pepper and represent the major yieldlimiting factors. In plants, specific transcription factors enhance the resistance to external stresses [22]. We previously identified seven stress-responsive CaNAC46 transcription factors in Capsicum species. In the current 


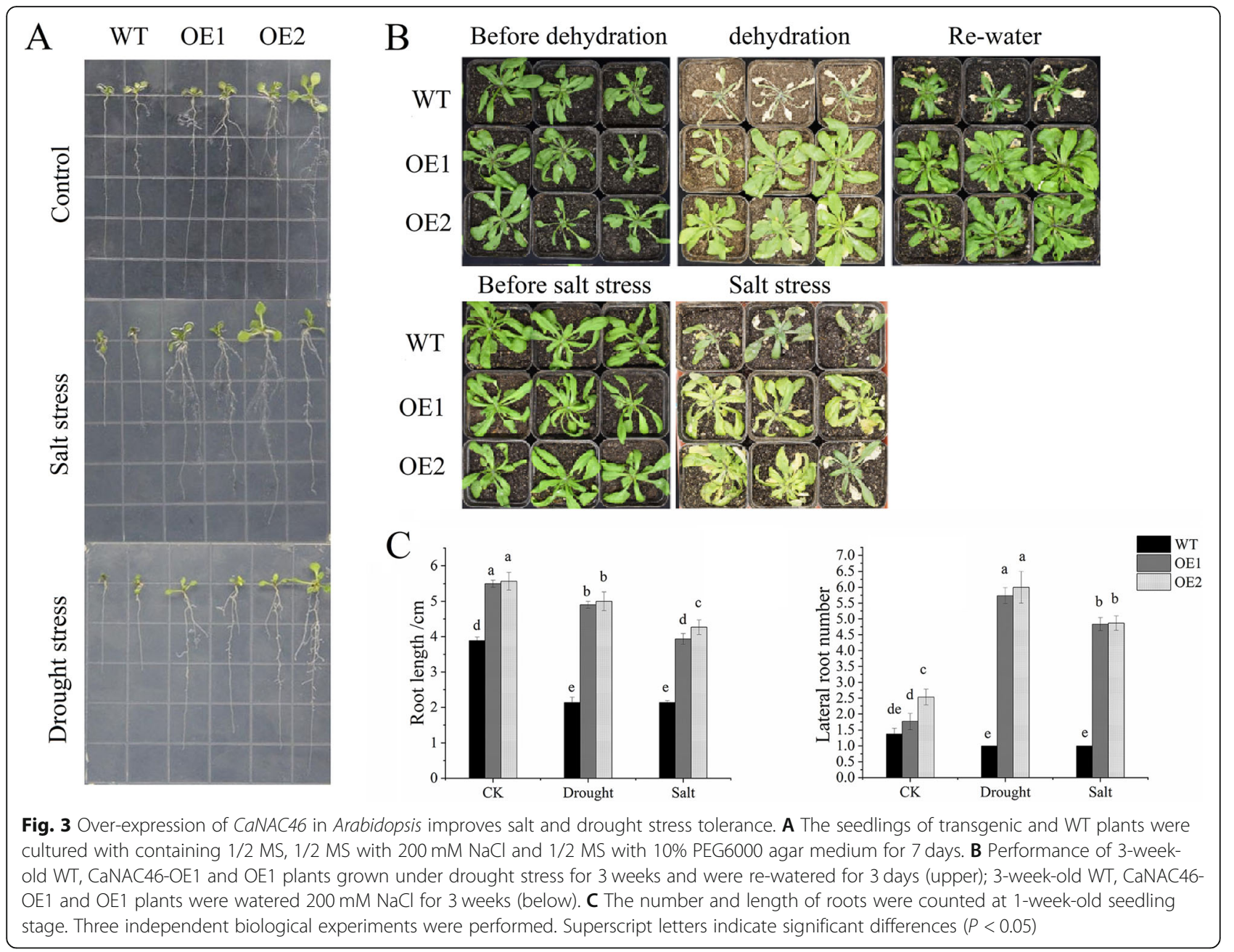

study, we proved that the overexpression of $\mathrm{CaNAC46}$ increases the tolerance to various abiotic stresses, including drought and salinity.

The CaNAC46 gene cloned from C. annuum 'Qingnong No. 2' consists of 921 bp that encode 306 amino acid residues. An analysis of the physicochemical properties revealed differences between CaNAC46 and NAC transcription factors from other plant species. This diversity may be relevant for future functional characterizations of NAC transcription factors. Additionally, CaNAC46 has three disordered amino acid regions, but their effects on the functions of the transcription factor remain to be determined. Ooka [8] classified NAC proteins into Groups I and II, with Group I divided into 14 subgroups. The NAC transcription factors in subgroups ATAF [23], AtNAC3 [24], and OsNAC3 [25] are involved in plant stress responses. The conserved sequences and phylogenetic relationships have been analyzed. The NAC transcription factors consist of a NAM subdomain with 160 amino acid residues as well as five other conserved subdomains (A, B, C, E, and D). In previous studies, the highly conserved subdomains A, C, and D were revealed to be important for NAC transcription factor functions. Subdomains $C$ and D are mainly involved in DNA binding. The significance of subdomain A results from the insertion of the dTph1 transposable element to form the NAM mutant [8]. Additionally, subdomains $B$ and $E$ vary among NAC proteins, suggesting they may be associated with the functional diversity of the NAC transcription factors [8]. Sequence comparisons with NAC family members confirmed that CaNAC46 belongs to the ATAF subfamily, and is most closely related to $A$. thaliana ATAF1.

Plant root system development is a relatively complex process, enabling plants to adapt to various environmental stresses. Salinity stress inhibits root meristem growth by decreasing auxin levels [26], whereas drought stress limits root growth and inhibits cytokinin production [27]. Root size and architecture influence the uptake of 


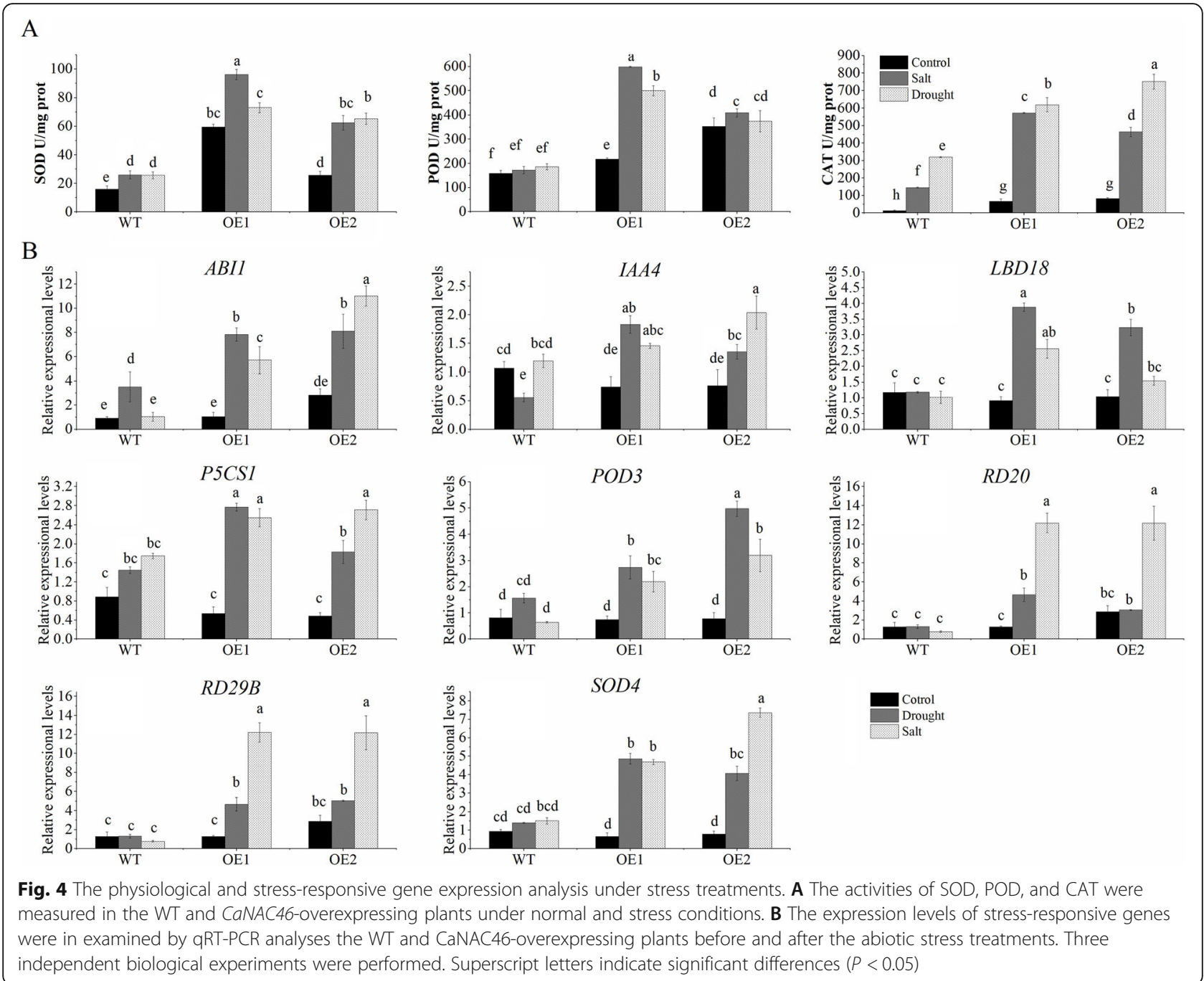

water and nutrients from the soil. In this study, CaNAC46-overexpressing plants produced longer roots and more lateral roots than WT plants following an exposure to drought and salinity stresses. Orthologs of $L B D 18$ in nonleguminous plants are required for lateral root development [28]. In A. thaliana, lateral root formation is regulated by indole-3-acetic acid (IAA) [29]. Our data indicated that $L B D 18$ and IAA4 expression levels were up-regulated in the CaNAC46-overexpressing plants in response to drought and high salt conditions. Accordingly, CaNAC46 may promote lateral root formation and root elongation by up-regulating $L B D 18$ and IAA4 expression to enhance the tolerance to drought and salinity stresses. The germination rate of CaNAC46-overexpressing lines was significantly lower than that of WT plants following the salt and drought treatments. These treatments also resulted in higher chlorophyll contents in the transgenic plants than in the WT controls.
In plants, proline is necessary for growth and development. Additionally, proline protects cells from damage by acting as a radical scavenger and an osmotic agent [15]. In the TRV2-CaNAC46 lines, the down-regulated expression of P5CS increased the sensitivity of the plants to salt and drought stress conditions. A previous study indicated that RD26 is a transcriptional activator mediating ABA-inducible gene expression in plants subjected to abiotic stress [30]. The rice NAC family transcription factors OsNAP and OsNAC2 respond to abiotic stresses via the ABA pathway [31, 32]. The NAC transcription factors may minimize the accumulation of ROS and regulate the expression of stress-related genes under drought and high salt conditions [33]. We speculate that the observed enhanced tolerance to abiotic stresses is mainly due to the up-regulated expression of stressresponsive genes, including $R D 20, R D 29 B, D R E B$, and $R D 22$. Following drought and salt treatments, $R D 20$ and $R D 29 B$ expression levels were up-regulated in the 


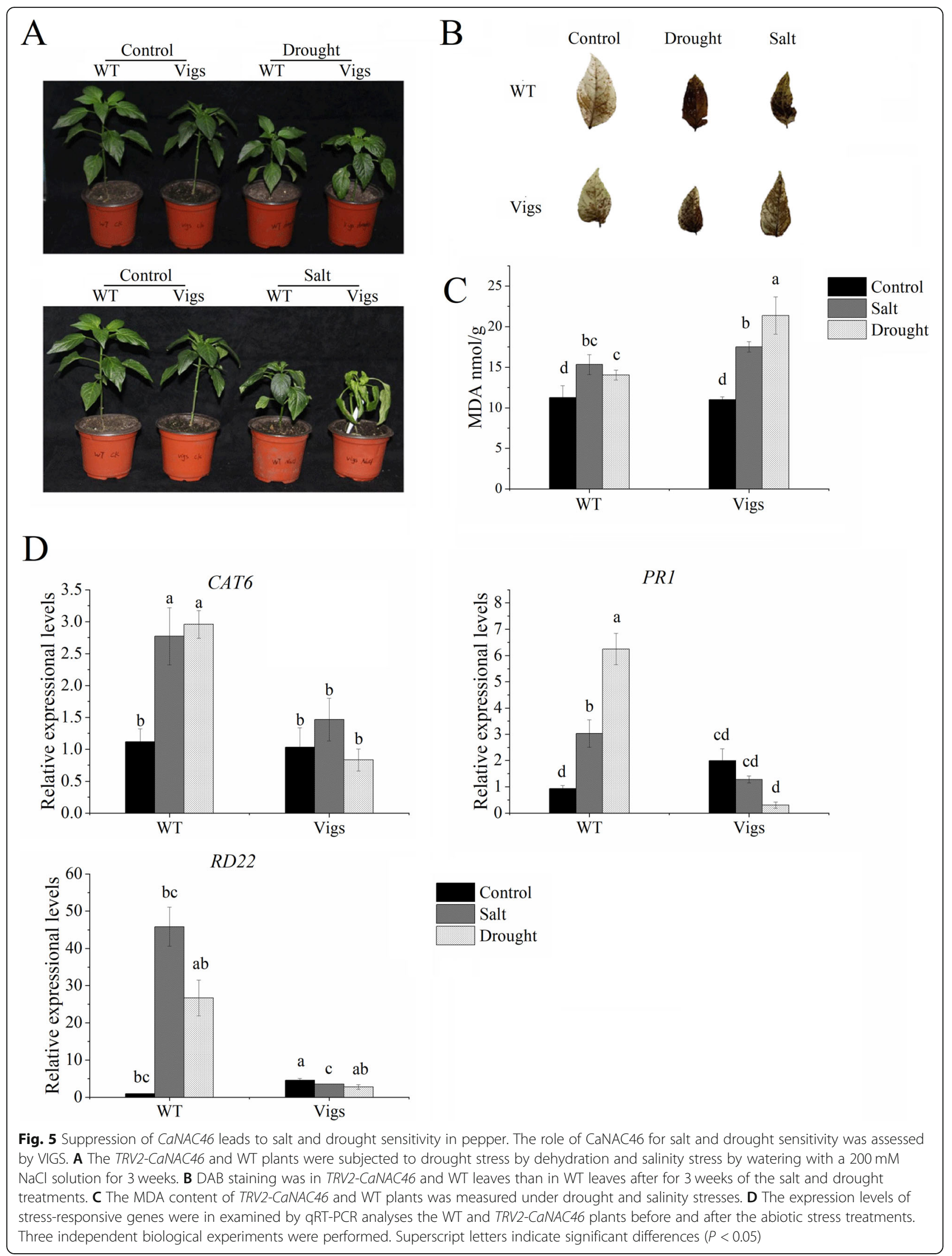


CaNAC46-overexpressing plants and DREB and RD22 expression levels were down-regulated in the TRV2CaNAC46 lines. The SOD, POD, and CAT activities were higher in the CaNAC46-overexpressing plants than in the control plants. Moreover, more MDA accumulated in the TRV2-CaNAC46 plants than in the WT plants. The DAB staining results proved that ROS accumulation was greater in the TRV2-CaNAC46 plants than in the control plants. The silencing of CaNAC46 expression via virus-induced gene silencing (VIGS) resulted in oxidative damage, as indicated by the accumulation of $\mathrm{H}_{2} \mathrm{O}_{2}$. These results suggested that CaNAC46 can activate the expression of stress signaling-related genes in response to abiotic stresses. In the CaNAC46-overexpressing plants, the expression levels of $A B I, P O D 3$, SOD2, P5CS1, and other genes involved in the ROSscavenging system were up-regulated to promote ROS scavenging and decrease stress-induced damages. The TRV2-CaNAC46 plants had relatively weak ROS activities, making them more sensitive to salt and drought stresses than the WT plants. Furthermore, CaNAC46 expression was correlated with CAT, POD, and SOD activities as well as with the transcription of most $S O D$ and $P O D$ genes. Thus, silencing CaNAC46 expression may be associated with increasing ROS accumulation and sensitivity to salt and drought stresses.

\section{Conclusion}

Our results suggest that CaNAC46 localized in the nucleus helps regulate abiotic stress responses. In this study, CaNAC46 expression was induced by high salinity, drought, heat, cold, ABA, SA, and MeJA treatments. The ectopic expression of CaNAC46 in A. thaliana resulted in enhanced root formation and stronger development following drought and salt stress treatments. Moreover, the expression levels of several stressresponsive genes related to ROS scavenging and lateral root development were significantly up-regulated by drought and salt stress. Additionally, CaNAC46-silenced lines exhibited relatively weak growth and increased ROS production (i.e., more intense $\mathrm{DAB}$ staining) in response to salt and drought stress treatments. Furthermore, the expression of ROS-related genes was downregulated in the CaNAC46-silenced lines. These findings imply that CaNAC46 may regulate salt and drought tolerance in pepper via its effects on ROS scavenging.

\section{Methods}

\section{Plant materials, growth conditions, and treatments}

Capsicum annuum L. var. 'Qingnong No. 2' was used as the plant material for various treatments and RNA isolation because of its tolerance to abiotic stresses. This cultivar was selected by researchers at Qingdao
Agricultural University (Qingdao, China), the Qingdao Seed Station, and Dezhou Academy of Agricultural Sciences. Additionally, it was approved by the Shandong Variety Examination and Approval Committee in 2015 (deposition number: 2015-057-1). Pepper seeds were preserved at Qingdao Agricultural University (Qingdao, China). After a 3-day germination, seedlings were grown in a growth chamber at $28^{\circ} \mathrm{C}$ with a 16-h light; 8-h dark cycle. All treatments were completed using 2-week-old seedlings. Regarding the simulated drought and saline conditions, seedling roots were treated with solutions containing 20\% (w/v) PEG 6000 and $200 \mathrm{mM} \mathrm{NaCl}$, respectively. For the cold and heat treatments, plants were transferred to growth chambers set to 4 and $37^{\circ} \mathrm{C}$, respectively. The phytohormone treatments involved immersing roots in an aqueous solution of $100 \mu \mathrm{M}$ ABA, MeJA, or SA. Leaves were collected at $0,2,4$, 8,12 , and $24 \mathrm{~h}$ after each treatment, immediately frozen in liquid nitrogen, ground to a powder, and then stored at $-80{ }^{\circ} \mathrm{C}$.

\section{Isolation and sequence analysis of CaNAC46}

Total RNA was extracted from leaves using the Plant Total RNA Kit (Yuanpinghao, Beijing, China). The RNA was then used as the template to synthesize cDNA with the PrimeScript RT Reagent Kit with gDNA Eraser (TAKARA, Dalian, China). The cDNA was diluted 20fold for the subsequent TA cloning and qRT-PCR. The CaNAC46 open reading frame was amplified by PCR using the following primers: forward: 5'-ATGATCAA AGGAATCGTTGGAAA-3'; reverse: 5'-CTAAGGTT TTTGCATGTATAGGA-3'. A multiple sequence alignment was completed with DNAMAN 6.0. The hydrophobicity, composition, and physicochemical properties of protein sequences were determined with the ExPASy online tools (http://www.expasy.org/). Additionally, a phylogenetic tree was constructed with MEGA 6.0.

\section{Quantitative real-time PCR}

Gene expression levels were analyzed by qRT-PCR, which was completed with the reverse transcribed cDNA and the LightCycler ${ }^{\circ} 480$ SYBR Green I Master kit (TAKARA). The reaction solution comprised $7 \mu \mathrm{L}$ $\mathrm{dd}_{2} \mathrm{O}, 10 \mu \mathrm{L}$ SYBR Green I Master mix, $1 \mu \mathrm{L}$ Forward Primer $(10 \mu \mathrm{mol} / \mathrm{L}), 1 \mu \mathrm{L}$ Reverse Primer $(10 \mu \mathrm{mol} / \mathrm{L})$, and $1 \mu \mathrm{L}$ cDNA. The PCR program was as follows: $95^{\circ} \mathrm{C}$ for $3 \mathrm{~min} ; 40 \mathrm{cycles}$ of $95^{\circ} \mathrm{C}$ for $5 \mathrm{~s}, 60^{\circ} \mathrm{C}$ for $20 \mathrm{~s}$, and $72{ }^{\circ} \mathrm{C}$ for $20 \mathrm{~s}$. This program was followed by a melting curve analysis. All reactions were performed in triplicate. Relative gene expression levels were determined based on the $2^{-\Delta \Delta \mathrm{Ct}}$ method. Primer sequences are listed in Table 1. 
Table 1 Primer sequences used for real-time qRT-PCR amplification

\begin{tabular}{|c|c|}
\hline Primer name & Primer sequence \\
\hline At-acting-F & TTCCTCATGCCATCCTCCGTCTT \\
\hline At-acting-R & CAGCGATACCTGAGAACATAGTG \\
\hline AtABl1-F & GGATTTCA CCGGGATCAGATTGG \\
\hline AtABI1-R & GTCACCGCAGTTAGCGACG AAG \\
\hline AtRD20-F & TTAGCTCCGGTCACCAGTCA \\
\hline AtABI1-R & TGTTCCATTCGGATGCTCTG \\
\hline AtP5CS1-F & GTTTCCTCAGCCGCCGATTITA \\
\hline AtP5CS1-R & GGAAACGCCACGTGTGGAAC \\
\hline AtRD29B-F & ATTCACCATCCAGAAGAAGAGCATC \\
\hline AtRD29B-R & ACTTCTGGGTCTTGCTCGTCA \\
\hline AtPOD3-F & CCAATCCGGAAACGGAAGTC \\
\hline AtPOD3-R & TCTGCATACTTCTTGACGAG \\
\hline AtSOD4-F & GAAGAACCTTGCTCCTTCCAG \\
\hline AtSOD4-R & GATTGGCAGTTGTGTCAACAAC \\
\hline AtIAA14-F & GCAGAGGAGGCAATGAGTAGTG \\
\hline AtIAA14-R & GAGCATCCAGTCACCATCTITG \\
\hline AtLBD18-F & GTCGCTCACATCTTTGCTCTTC \\
\hline AtLBD18-R & AGGTAGCTCTAGTGATGCCAAATG \\
\hline CaNAC46-F & ATGATCAAAGGAATCGTTGGAAA \\
\hline CaNAC46-R & CTAAGGTIITGCATGTATAGGA \\
\hline CaPR1-F & ACTTGCAATTATGATCCACC \\
\hline CaPR1-R & ACTCCAGTTACTGCACCAT \\
\hline CaRD22-F & AGTAACTCTGGCAGTGGCAC \\
\hline CaRD22-R & GTAGACTGGCGGCTTTCCTT \\
\hline Ca-actin-F & GGTGACGAGGCTCAATCCAA \\
\hline Ca-actin-R & CTCTGGAGCCACACGAAGTT \\
\hline CaCAT6-F & TCTTGTTATTCGCGGGCCTT \\
\hline CaCAT6-R & TGAAGGGGACAAACCAACCC \\
\hline
\end{tabular}

\section{Subcellular localization of CaNAC46}

The CaNAC46 coding sequence was amplified by PCR and inserted into the SpeI and $A s c$ I sites of the modified pMDC83 vector with the ClonExpress II One Step Cloning Kit (Vazyme, Nanjing, China). After sequencing to confirm the accuracy of the inserted fragment, the recombinant plasmid and the empty pMDC83 vector (i.e., GFP alone) were introduced into tobacco cells according to an Agrobacterium tumefaciens-mediated method [34]. After a 24-h incubation in darkness, GFP fluorescence in transformed tobacco cells was observed with the FluoView $^{\text {ma }}$ FV300 confocal microscope (Olympus, Japan).

\section{Generation of CaNAC46-overexpressing transgenic Arabidopsis thaliana plants}

The CaNAC46 coding sequence was amplified by PCR and cloned into the $\mathrm{Xba \textrm {I }}$ sites of the pBI121 vector for the subsequent expression under the control of the cauliflower mosaic virus (CaMV) $35 \mathrm{~S}$ promoter. The accuracy of the resulting construct was confirmed by sequencing, after which the pBI121-CaNAC46 recombinant plasmid was inserted into A. thaliana plants according to a floral dip method involving $A$. tumefaciens strain GV3101. Putative transgenic plants were screened on MS medium containing kanamycin and identified by PCR. Four $\mathrm{T}_{3}$ homozygous CaNAC46-overexpressing transgenic plants were selected for further analyses.

\section{Performance of CaNAC46-overexpressing Arabidopsis thaliana plants under saline and drought conditions}

Homozygous CaNAC46-overexpressing lines from the $\mathrm{T}_{3}$ generation underwent a phenotypic analysis. The WT and transgenic lines were initially cultured on $1 / 2 \mathrm{MS}$ agar medium at $22{ }^{\circ} \mathrm{C}$ with a 16 -h light/8-h dark cycle for 7 days. The seedlings were then transferred to $1 / 2$ MS agar medium supplemented with $200 \mathrm{mM} \mathrm{NaCl}$ or 10\% PEG 6000. The seedlings were incubated for 1 week. Additionally, to investigate the drought tolerance of older plants, seedlings were grown in soil under normal conditions for 4 weeks, after which watering was withheld for 2 weeks and then resumed for 3 days. To evaluate the salt stress tolerance of older plants, WT and transgenic plants were grown in soil under normal conditions for 4 weeks, after which they were irrigated three times (4-day intervals) with a $200 \mathrm{mM}$ $\mathrm{NaCl}$ solution.

\section{Malondialdehyde content and antioxidant enzyme activity measurements}

The MDA contents as well as the SOD, POD, and CAT activities were quantified with commercial detection kits (Jiancheng Bioengineering Institute, Nanjing, China). The chlorophyll content was measured with a hand-held chlorophyll meter.

\section{Virus-induced gene silencing}

A VIGS experiment was performed with pTRV1 and pTRV2 vectors. The CaNAC46 sequence was inserted into the pTRV2 vector. The sequence to be silenced was determined using the SGN VIGS Tool (https://vigs.solgenomics.net/), and was amplified with the following primers (TRV-CaNAC46): forward: 5'-TTGGTGCAGC ATTATTTGTGC-3'; reverse: 5' -ATTTTTGGTTTTAT TTCATCCTCAA-3'. Agrobacterium tumefaciens cells containing pTRV1 were mixed with $A$. tumefaciens cells transformed with pTRV2-CaNAC46. The combined bacterial solution was infiltrated into the lower leaves of pepper plants. At 3 weeks post-infiltration, the silencing of CaNAC46 was assessed via physiological measurements and histochemical assays. 


\section{Statistical analysis}

Bioassay results were evaluated with Origin and DPS. The significance of the differences in the data was determined with Student's $t$-test $(P<0.05)$.

\section{Supplementary Information}

The online version contains supplementary material available at https://doi. org/10.1186/s12870-020-02764-y.

Additional file 1: Table S1. A comparison of physical and chemical characterization of CaNAC46 transcription factors with its homolog NAC in other model plants.

Additional file 2: Figure S1. The germination rates of WT and CaNAC46-overexpressing plants under the simulated drought and salt conditions.

Additional file 3: Figure S2. The expression of CaNAC46 gene in WT and OE plants by RT-PCR (1-4 represent WT plants; $5-8$ represent OE plants).

Additional file 4: Figure S3. The expression of CaNAC46 gene in WT and TRV-CaNAC46 plants by RT-PCR (A, 1-4 represent WT plants, 5-8 represent TRV- CaNAC46 plants) and by qRT-PCR (B, 1 represent WT, 2 represent TRV-CaNAC46 plants).

\section{Abbreviations}

ABA: Abscisic acid; ACC: 1-aminocyclopropane-1-carboxylic-acid; CaMV: Cauliflower mosaic virus; DAB: Diaminobezidine; IAA: Indole-3-acetic acid; LBD: Lateral organ boundaries domain; MDA: Malondialdehyde; MeJA: Methyl jasmonate; NAC: NAM, ATAF1/ATAF2, and CUC2; P5CS: $\Delta^{1}$ pyrroline-5-carboxylate synthetase; PEG: Polyethylene glycol; PLD: Phospholipase D; PR: Pathogenesis related; qRT-PCR: Quantitative reverse transcription-polymerase chain reaction; $\mathrm{RD}$ : Response to dehydration; ROS: Reactive oxygen species; SA: Salicylic acid; VIGS: Virusinduced gene silencing; WT: Wild type

\section{Acknowledgments}

We thank the Central Laboratory of Qingdao Agricultural University for the FluoView ${ }^{\mathrm{TM}}$ FV300 confocal microscope. We thank all of the teachers and students of the Key Laboratory of Horticultural Plant Genetic Improvement and Breeding of Qingdao for providing guidance and assistance. We thank Liwen Bianji, Edanz Editing China (www.liwenbianji.cn/ac) for editing the English text of a draft of this manuscript.

\section{Authors' contributions}

JM, DL and LYW conceived, designed and wrote the manuscript. LYW analyzed data. LYW, JXD and YW performed all experiments. Both authors read and approved the final manuscript.

\section{Funding}

The work was supported by Central Government Guides Local Science Development Project, China (YDZX20193700004393) and Agricultural Superior Seed Engineering Program of Shandong, China (2016LZGC011). The funders had no role in the design of the study and collection, analysis, and interpretation of data and in writing the manuscript.

\section{Availability of data and materials}

All data generated or analyzed during this study are included in this article (and its supplementary information files) or are available from the corresponding author on reasonable request. Sequence data from this article can be found in Arabidopsis Information Resource (https://www.arabidopsis. org/) under the following accession numbers: ATAF1 (At1g01720), ATAF2 (AT5G08790), ANAC036 (At2g17040), ANAC098 (At5g53950). The sequences of OsNAC005 (LOC_Os11g08210), OsNAC006 (LOC_Os01g66120), ONAC072 (LOC_Os09g32260) and ONAC022 (LOC_Os03g04070) are available in Genome Annotation Batch Download of Rice Genome Annotation Project (RGAP: http://rice.plantbiology.msu.edu/downloads_gad.shtml). In addition, the sequences of SINAC053 (Solyc06g060230), SINAC048 (Solyc05g055470), SINAC067 (Solyc07g053590) and SINAC091 (Solyc00g008000) are available in locus search of Sol Genomics Network (SGN: https://solgenomics.net/search/ locus) database, and CaNAC52 (Capana05g002477), CaNAC71 (Capana07g002159), CaNAC101 (Capana12g002456), CaNAC20 (Capana02g000302), CaNAC46 (Capana05g000569) can be downloaded from the Pepper Genome Platform (PGP: http://peppergenome.snu.ac.kr/ download.php). StNAC53 (CAC42087.1) is available in National Center for Biotechnology Information (NCBI, https://www.ncbi.nlm.nih.gov/).

\section{Ethics approval and consent to participate}

Not applicable.

\section{Consent for publication}

Not applicable.

\section{Competing interests}

The authors declare that there are no competing interests.

Received: 19 March 2020 Accepted: 1 December 2020

Published online: 06 January 2021

\section{References}

1. Yao MH, Yin YX, Wang F, Ning LI, Jiao CH, Zhao JY. Current situation and countermeasures of breeding for processing pepper in China. Hubei Agric Sci. 2017;17(2):323-8 (in Chinese).

2. Adams MJ, Ahuja KDK, Geraghty DP. Effect of capsaicin and dihydrocapsaicin on in vitro blood coagulation and platelet aggregation. Thromb Res. 2009;124(6):721-3.

3. Nakashima K, Takasaki H, Mizoi J, Shinozaki K, Yamaguchi-Shinozaki K. NAC transcription factors in plant abiotic stress responses. Biochim Biophys Acta. 2012:1819(2):97-103.

4. Sakuma Y, Maruyama K, Qin F, Osakabe Y, Yamaguchi-Shinozaki K. Dual function of an Arabidopsis transcription factor DREB2A in water-stressresponsive and heat-stress-responsive gene expression. Proc Natl Acad Sci U S A. 2007;103(49):18822-7.

5. Khattab HI, Emam MA, Emam MM, Helal NM, Mohamed MR. Effect of selenium and silicon on transcription factors NAC5 and DREB2A involved in drought-responsive gene expression in rice. Biol Plant. 2014;58(2):265-73.

6. Kang M, Kim S, Kim HJ, Shrestha P, Yun J-H, Phee B-K, Lee W, Nam HG, Chang I. The C-domain of the NAC transcription factor ANAC019 is necessary for $\mathrm{pH}$-tuned DNA binding through a Histidine switch in the $\mathrm{N}$ domain. Cell Rep. 2018;22(5):1141-50.

7. Zhong R, Lee C, Zhou J, McCarthy R, Zheng H. A battery of transcription factors involved in the regulation of secondary cell wall biosynthesis in Arabidopsis. Plant Cell. 2008;20(10):2763-82.

8. Ooka H, Satoh K, Doi K, Nagata T, Otomo Y, Murakami K, Matsubara K, Osato N, Kawai J, Carninci P, Hayashizaki Y, Suzuki K, Kojima K, Takahara Y, Yamamoto K, Kikuchi S. Comprehensive analysis of NAC family genes in Oryza sativa and Arabidopsis thaliana. DNA Res. 2003;10(6):239-47.

9. Le DT, Nishiyama R, Watanabe Y, Mochida K, Yamaguchi-Shinozaki K, Shinozaki K, Tran LS. Genome-wide survey and expression analysis of the plant-specific NAC transcription factor family in soybean during development and dehydration stress. DNA Res. 2011;18(4):263-76.

10. Liu G, Li X, Jin S, Liu X, Zhu L, Nie Y, Zhang X. Overexpression of rice NAC gene SNAC1 improves drought and salt tolerance by enhancing root development and reducing transpiration rate in transgenic cotton. PLoS One. 2014;9(1):e86895.

11. Oh SK, Lee S, Yu SH, Choi DJ. Expression of a novel NAC domaincontaining transcription factor (CaNAC1) is preferentially associated with incompatible interactions between chili pepper and pathogens. Planta. 2005;222(5):876-87.

12. X-m H, H-f Z, S-y L, Wang X-k, Y-m Z, Y-c M. Luo D, Chen R-g: the NAC transcription factor CaNAC064 is a regulator of cold stress tolerance in peppers. Plant Sci. 2020;291:110346-55.

13. Diao W, Snyder JC, Wang S, Liu J, Pan B, Guo G, Ge W. Genome-wide analyses of the NAC transcription factor gene family in pepper (Capsicum annuum L.): chromosome location, phylogeny, structure, expression patterns, Cis-elements in the promoter, and interaction network. Int J Mol Sci. 2018;19(4):1048-61.

14. Guo WL, Wang SB, Chen RG, Chen BH, Du XH, Yin YX, Gong ZH, Zhang YY. Characterization and expression profile of CaNAC2 pepper gene. Front Plant Sci. 2015;6:755. 
15. He L, Shi X, Wang Y, Guo Y, Yang K, Wang Y. Arabidopsis ANAC069 binds to $\mathrm{C}[\mathrm{A} / \mathrm{G}] \mathrm{CG}[\mathrm{T} / \mathrm{G}]$ sequences to negatively regulate salt and osmotic stress tolerance. Plant Mol Biol. 2017;93(4-5):369-87.

16. Fang $Y$, Liao $K, D u H, X u Y$, Song $H$, Li X, Xiong L. A stress-responsive NAC transcription factor SNAC3 confers heat and drought tolerance through modulation of reactive oxygen species in rice. J Exp Bot. 2015; 66(21):6803-17.

17. Hu H, Dai M, Yao J, Xiao B, Li X, Zhang Q, Xiong L. Overexpressing a NAM, ATAF, and CUC (NAC) transcription factor enhances drought resistance and salt tolerance in rice. Proc Natl Acad Sci U S A. 2006;103(35):12987-92.

18. Mauch-Mani B, Flors V. The ATAF1 transcription factor: at the convergence point of ABA-dependent plant defense against biotic and abiotic stresses. Cell Res. 2009;19(12):1322-3.

19. Takasaki H, Maruyama K, Kidokoro S, Ito Y, Fujita Y, Shinozaki K, YamaguchiShinozaki K, Nakashima K. The abiotic stress-responsive NAC-type transcription factor OsNAC5 regulates stress-inducible genes and stress tolerance in rice. Mol Gen Genomics. 2010;284(3):173-83.

20. Nakashima K, Tran LSP, Dong VN, Fujita M, Yamaguchi-Shinozaki K. Functional analysis of a NAC-type transcription factor OsNAC6 involved in abiotic and biotic stress-responsive gene expression in rice. Plant J. 2010;51(4):617-30.

21. Tran H. High throughput profiling of transcription factors involved in soybean root growth under water deficit. Columbia: University of MissouriColumbia; 2009

22. Erpen L, Devi HS, Grosser JW, Dutt M. Potential use of the DREB/ERF, MYB, NAC and WRKY transcription factors to improve abiotic and biotic stress in transgenic plants. Plant Cell Tissue Organ Cult An Int J in Vitro Cult. 2018; 132(1):1-25

23. Lu PL, Chen N-Z, An R, Su Z, Qi B-S, Ren F, Chen J, Wang X-C. A novel drought-inducible gene, ATAF1, encodes a NAC family protein that negatively regulates the expression of stress-responsive genes inArabidopsis. Plant Mol Biol. 2007;63(2):289-305.

24. Al-Abdallat AM, Ali-Sheikh-Omar MA, Alnemer LM. Overexpression of twoATNAC3-related genes improves drought and salt tolerance in tomato (Solanum lycopersicum L.). Plant Cell Tissue Organ Cult. 2015; 120(3):989-1001.

25. Zhou XG, Chen XJ. Identification a new NAC transcription factor OsNAC3 in rice. Acta Phytopathologica Sin. 2018:48(1):61-9 (in Chinese).

26. Liu W, Li R-J, Han TT, Cai W, Fu ZW, Lu Y-TJPP. Salt stress reduces root meristem size by nitric oxide-mediated modulation of Auxin accumulation and signaling in Arabidopsis. Plant Physiol. 2015;168(1):343-56.

27. Xu Y, Burgess $P$, Zhang $X$, Huang B. Enhancing cytokinin synthesis by overexpressing ipt alleviated drought inhibition of root growth through activating ROS-scavenging systems in Agrostis stolonifera. J Exp Bot. 2016; 67(6):1979-92

28. Lee HW, Kim JJP, Physiology C. EXPANSINA17 up-regulated by LBD18/ASL20 promotes lateral root formation during the Auxin response. Plant Cell Physiol. 2013;54(10):1600-11.

29. Goh T, Kasahara H, Mimura T, Kamiya Y, Fukaki HJPTRSLBBS. Multiple AUX IAA-ARF modules regulate lateral root formation: the role of Arabidopsis SHY2/IAA3-mediated auxin signalling. Philos Trans R Soc B Biol Sci. 2012; 367(1595):1461-8.

30. Fujita M, Fujita Y, Maruyama K, Seki M, Hiratsu K, Ohme-Takagi M, Tran L-SP, Yamaguchi-Shinozaki K. A dehydration-induced NAC protein, RD26, is involved in a novel ABA-dependent stress-signaling pathway. Plant J. 2004; 39(6):863-76.

31. Hsin Lu S, Wang FH, Li J, Li J, Lv B, Ma H, Chen X, Wang Y, Luo Q, Ming F. The NAC family transcription factor OsNAP confers abiotic stress response through the ABA pathway. Plant Cell Physiol. 2014;55(3):604-19.

32. Shen J, Lv B, Luo L, He J, Mao C, Xi D, Ming F. The NAC-type transcription factor OsNAC2 regulates ABA-dependent genes and abiotic stress tolerance in rice. Sci Rep. 2017;7:40641-56.

33. Yuan $X$, Wang H, Cai J, Bi Y, Biology FSJBP. Rice NAC transcription factor ONAC066 functions as a positive regulator of drought and oxidative stress response. BMC Plant Biol. 2019;19(1):278-86.

34. Kim CK, Chung JD, Park SH, Burrell AM, Kamo KK, Byrne DH. Agrobacterium tumefaciens-mediated transformation of Rosa hybridausing the green fluorescent protein (GFP) gene. Plant Cell Tissue Organ Cult. 2004;78(2):107-11.

\section{Publisher's Note}

Springer Nature remains neutral with regard to jurisdictional claims in published maps and institutional affiliations.

Ready to submit your research? Choose BMC and benefit from:

- fast, convenient online submission

- thorough peer review by experienced researchers in your field

- rapid publication on acceptance

- support for research data, including large and complex data types

- gold Open Access which fosters wider collaboration and increased citations

- maximum visibility for your research: over $100 \mathrm{M}$ website views per year

At BMC, research is always in progress.

Learn more biomedcentral.com/submissions 